\title{
First results from the FPGA/NIOS Adaptive FIR Filter Using Linear Prediction Implemented in the AERA Radio Stations to Reduce Narrow Band RFI for Radio Detection of Cosmic Rays
}

\author{
Zbigniew Szadkowski, Member, IEEE, D. Głas, C. Timmermans, T. Wijnen, \\ for the Pierre Auger Collaboration
}

\begin{abstract}
The FPGA/NIOS ${ }^{\circledR}$ FIR filter based on linear prediction (LP) to suppress radio frequency interference (RFI) has been installed in several radio stations in the Auger Engineering Radio Array (AERA) experiment. AERA observes coherent radio emission from extensive air showers induced by ultra-high-energy cosmic rays to make a detailed study of the development of the electromagnetic part of air showers. Radio signals provide complementary information to that obtained from Auger surface detectors, which are predominantly sensitive to the particle content of an air shower at the surface. The radio signals from air showers are caused by the coherent emission due to geomagnetic and charge-excess processes. These emissions can be observed in the frequency band between $10-100 \mathrm{MHz}$. However, this frequency range is significantly contaminated by narrow-band RFI and other human-made distortions. A FIR filter implemented in the FPGA logic segment of the front-end electronics of a radio sensor significantly improves the signal-to-noise ratio. Theoretical calculations show a high efficiency of this filter for mono-carrier as well as for standard FM radio contaminations. The laboratory tests, performed on the Altera ${ }^{\circledR}$ Cyclone $^{\circledR}$ V DK-DEV-5CEA7N development kit confirmed the theoretical expectations.

In this paper we present first results of the efficiency of the adaptive LP FIR filter, deployed in real AERA station on pampas, with a comparison to the currently used IIR notch filter with constant coefficients. The coefficients for the linear predictor are dynamically refreshed and calculated in a Voipac PXA270M ARM processor, which is implemented on a daughter-board placed in the same digital unit as the FPGA. The laboratory tests confirms the stability of the filter. Using constant LP coefficients the suppression efficiency remains the same for hours, which corresponds to more than $10^{12}$ clock cycles. We compared in real conditions several variants of the LP FIR filter with various lengths and various coefficients widths (due to fixed-point representations in the FPGA logic) with the aim to minimize the power consumption for the radio station while keeping sufficient accuracy for noise reduction.
\end{abstract}

The laboratory and real condition tests provide data to optimize the RFI cleaning for the next generation of the AERA FrontEnd based on Cyclone ${ }^{\circledR}$ V with Hardcore Processor System (HPS) and System on Chip (SoC).

Manuscript received June 03, 2014

Zbigniew Szadkowski is with the University of Łódź, Department of Physics and Applied Informatics, Faculty of High-Energy Astrophysics, 90-236 Łódź, Poland, (e-mail: zszadkow @kfd2.phys.uni.lodz.pl, phone: +48 4263556 59).

Dariusz Głas is the PhD student with the University of Łódź, Department of Physics and Applied Informatics, Faculty of High-Energy Astrophysics, 90-236 Łódź, Poland,

Charles Timmermans, Thei Wijnen are with Nikhef, Science Park, Amsterdam, Netherlands, and IMAPP, Radboud University Nijmegen, Netherlands

\section{INTRODUCTION}

$\mathbf{R}$ ECENTLY radio detection of cosmic-ray air showers relives a renaissance, mainly thanks to a huge progress of the powerful digital signal processing techniques in experiments such as LOPES [1], CODALEMA [2] or the Auger Engineering Radio Array (AERA) [3], which is situated within the Pierre Auger Observatory (PAO) [4].

Results from the Pierre Auger Observatory, point to the need for very large aperture detection systems for ultra-high energy cosmic rays. With its nearly $100 \%$ duty cycle, its high angular resolution, and its sensitivity to the longitudinal air-shower evolution, the radio technique is particularly well-suited for detection of ultra-high energy cosmic rays (UHECRs) in large-scale arrays. AERA has been enlarged to 124 radio detector stations (RDSs), covering an area of $6.5 \mathrm{~km}^{2}$ therefore allowing the detection of UHECRs.

Since the 1960s we know that the radio emission from air showers is strongly correlated with the local geomagnetic field [5]. In addition to the geomagnetic effect that can be described macroscopically [6], Askaryan [7] [8] predicted that there should be an emission component related to the timevariation of the negative net charge excess in air showers. Both [1] and [2] confirmed these effects. Using the AERA setup, the Auger collaboration quantified the relative strength of these effects [9] The observation of air showers with radio-detection techniques can be done at almost all times. Moreover, radio signals are sensitive to the development of the electromagnetic component of particle showers in the atmosphere of the Earth. In the last 10 years the radio-detection technique in the $\mathrm{MHz}$ region has been revived and the present radio-detector arrays for cosmic-ray research are equipped with low-noise and highrate digital samplers. Simultaneously, the number of stations within these arrays has grown from less than ten to more than one thousand. The question to be addressed in the VHF band (MHz-range) is not whether extensive air showers emit radiation. At this moment the main question to answer is: can we use radio signals to determine the primary energy, the arrival direction, and the mass of cosmic rays with accuracies which are equal to or better than those obtained by other techniques? And if yes, can we build for an affordable price a huge surface-detector array based on the radio-detection technique? 

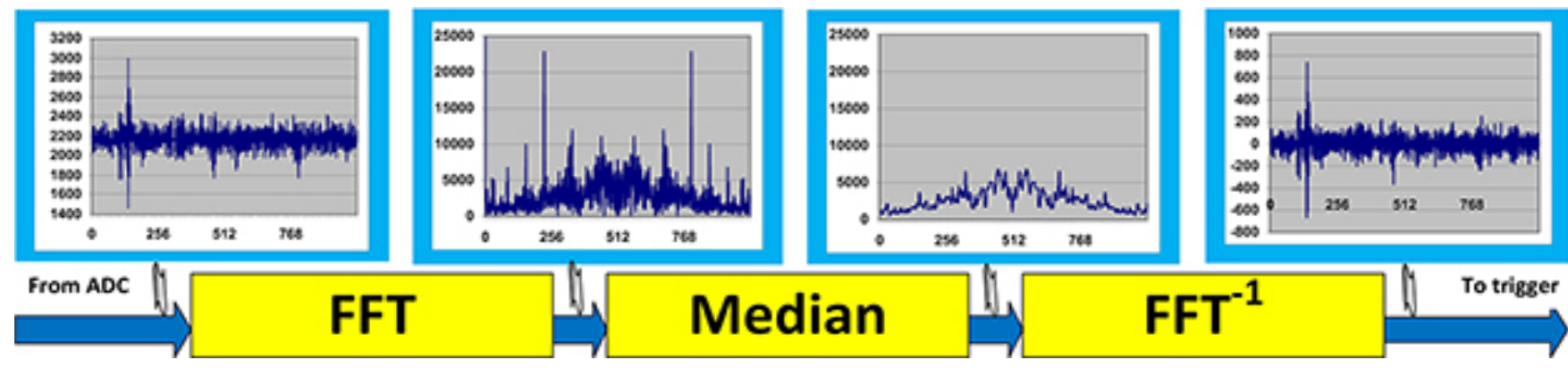

Median

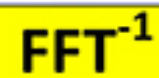

Fig. 1. A diagram showing a (FFT + Median filter + iFFT) chain cleaning the signal from the RFI contamination. The 1st graph shows the ADC input as unsigned data with an offset of ca. 2300 ADC-counts, the 2nd - the absolute values of FFT coefficients in the frequency domain, the 3rd - FFT coefficients decontamined by the median filter and 4th - signal converted back to the time domain. Additionally, the 0th FFT coefficient has been zeroed. Thus, the cleaned signal in the time domain is represented as signed data without the offset. The amplitude of the signal remains roughly the same and the noise is considerably reduced.

\section{RFI SUPPRESSION FOR REAL AERA DATA}

Triggering directly on the radio signal of the air showers (instead of using particle detectors as a trigger) poses some challenges for the data acquisition, due to man-made radiofrequency interference (RFI). The continuous background level is set by the radio emission from the Galactic plane, but any man-made narrow band transmitters add to the level above which one must detect air-shower pulses. Additionally, man-made pulsed RFI (from sparking electrical equipment, airplanes, etc.) can mimic the short pulsed signal from cosmic rays. Since the bandwidth and computational resources at each triggering level are limited, one of the technical focuses for the first stage of the array has been to develop various methods to reject RFI in order to minimize efficiency losses from bandwidth saturation.

The energy threshold of radio detection of cosmic rays is limited by the considerable radio background and noise. The very high level of RFI in the FM and short wave band has to be eliminated by a band pass filter. Within the remaining receiver bandwidth of 30 to $80 \mathrm{MHz}$ the noise at the quiet-rural environment of the Pierre Auger Observatory is dominated by the frequency dependent galactic noise [10] with noise temperatures of $5000 \mathrm{~K}$ at $60 \mathrm{MHz}$

In addition to the galactic noise, there is a human made background. This background consists of continuous signals, as from a few radio and TV stations, and transients produced by machines. Without an effective trigger, a stable and low level energy threshold is not guaranteed. Furthermore, the data rate for communication of the triggered data to the central DAQ would exceed the available bandwidth.

\section{A. $F F T+$ Median filter $+i F F T$}

For self-triggered measurements, the data will be digitized and processed in real time by a powerful FPGA chip. The narrow peaks in the frequency domain due to radio frequency interferences have to be strongly suppressed before building a trigger. These peaks are removed in several stations, digitizing at $180 \mathrm{MHz}$, using a median filter. The filter works in the frequency domain using the Fast Fourier Transform (FFT) routine provided by Altera. Furthermore, the phase of the signal deformed by the steep band pass filter is reconstructed by a deconvolution in the frequency domain. The median FPGA filter eliminates mono-frequent carriers, but broadband radio pulses from cosmic showers are not affected. After a second inverse FFT, signals are converted back to the time domain. This chain of the digital signal processing strongly enhances the signal to noise ratio, and thus improves the radio pulse detection sensitivity (Fig. 1).

In order to suppress the strong man-made radio carrier signals below $30 \mathrm{MHz}$ and above $80 \mathrm{MHz}$ and to fulfill the Nyquist theorem, the signals of the antennas are filtered using an analogue band filter before being sampled by the ADCs. The filters with the constant transfer function $H(f)$ are of high order with a non-constant group delay, thus leading to a dispersion and decreasing the amplitude of the input signals $S(f)$. In a frequency domain, the resulting output signal $P(f)$ can simply be calculated using (1) :

$$
P(f)=H(f) \cdot S(f)
$$

Knowing $H(f)$ from measurements, it is now easy to get back $S(f)$ by inverting (1). This operation can be implemented into the FPGA by placing a complex multiplication unit directly after the FFT engine which multiplies the output data of the engine with the precomputed coefficients of $1 / H(f)$ stored in a RAM. After transforming the signal back into the time domain, the amplitude of the de-convoluted signal increases by about $20 \%$ compared to the input signal. Since the galactic and electronic noise is completely uncorrelated, the $\mathrm{S} / \mathrm{N}$ ratio increases by the same amount.

Aliasing appears when converted pulses are located close to a border of converted blocks. It manifests by a spurious contribution in the opposite border of the block and in the neighboring block as well. This effect may cause spurious triggers and has to be eliminated.

The problems can only be solved, without introducing dead time between the blocks, by using an overlapping routine. Therefore the filter engine must run in another clock domain with higher frequency $(210 \mathrm{MHz}$ instead of $180 \mathrm{MHz}$ ). Nevertheless, it requires additional resources e.g. FILO (First In Last Out) memory based procedure to inverse in time a sequence of samples. The FFT approach is generally very power consuming. This is a factor for a system supplied from solar panels. 


\section{B. IIR notch filter}

As stated earlier, before triggering on a radio pulse it is advantageous to increase the signal-to-noise ratio by filtering out any narrow band transmitters from the digitized antenna signals. In stations digitizing at $200 \mathrm{MHz}$, this is accomplished in a computationally efficient manner by using a series of infinite-impulse response (IIR) notch filters in a Cyclone ${ }^{\circledR}{ }_{-}$ IV FPGA. The IIR filters operate on the time-domain signal, and the output of the filter $y_{i}$ is a linear combination of input samples $x_{j}$ and delayed feedback output samples $y_{j}$ from the filter:

$$
\begin{aligned}
y_{i}=x_{i} & -\left(2 \cos \omega_{N} \cdot x_{i-1}\right)+x_{i-2}+ \\
& +\left(2 r \cos \omega_{N} \cdot y_{i-1}\right)-\left(r^{2} \cdot y_{i-2}\right)
\end{aligned}
$$

The normalized filter frequency $\omega_{N}=2 \pi f_{N} / f_{S}$ is given by the notch frequency $f_{N}$ and the sampling frequency $f_{S}$. The width parameter, $r$, is provided with a value strictly between 0 and 1 , with higher values giving a narrower response function. For a narrow transmitter a typical value is $r=0.99$. A complication in the implementation of the IIR filters in high-frequency FPGAs arises from the fact that one cannot arbitrarily pipeline the feedback computation. We have resolved this by using the scattered look-ahead pipelining technique [11], which increases the filter complexity but allows more time for computation in the FPGA.

The coefficients of the $x_{j}$ and $y_{j}$ in Eq. 2 can be precomputed for the desired notch frequencies, converted to a fixed-point representation and loaded into the FPGA at runtime. The current design allows for four independent tunable notch filters for each polarization direction and is applied on a Cyclone ${ }^{\circledR}$ III, IV and V FPGA. It has been operating in the field for several years and successfully decreased and stabilized the threshold settings.

\section{Linear predictor}

Linear prediction is a mathematical operation where future values of a discrete-time signal are estimated as a linear function of previous samples [12]. This method is widely used in audio signal processing and speech processing for representing the spectral envelope of a digital signal of speech in compressed form, using the information of a linear predictive model [13]. With the advent of faster signal processing techniques in FPGAs it is now possible to apply similar techniques to the real-time processing of radio signals in the $10-100 \mathrm{MHz}$ region [14].

In the LP method the covariances for 1024 ADC samples can be calculated in the FPGA fast logic block. Either the

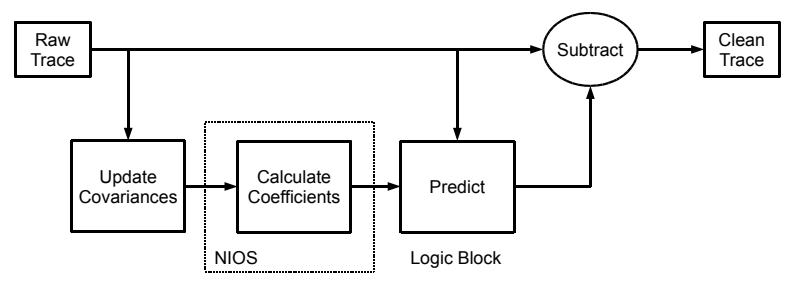

Fig. 2. The data flow of the FIR filter based on the LP method
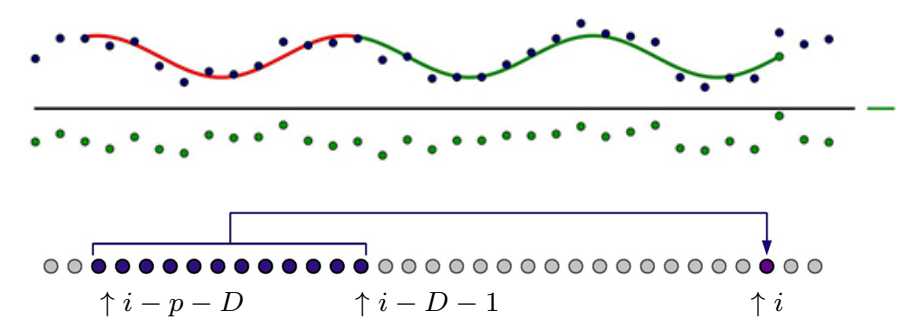

Fig. 3. An illustration of the method. The sine wave represents the signal that is 'fitted' (although in actuality no sinusoidal fit is performed like this) where sample number $i$ is predicted by using the samples $i-p-D$ to $i-D$. The predicted values are then subtracted from the original values as illustrated by the green dots below the horizontal bar with the minus sign, reducing the variance of the signal.

NIOS ${ }^{\circledR}$ processor, or the external ARM-processor, solves the matrix of 32 or 64 linear equations and provides coefficients needed for the FIR filter. The calculated coefficients are next transferred to the fast logic block, updating appropriate registers. They are used as the FIR coefficients in the ADC data filtering. Finally, the predicted and delayed data (expected background) are subtracted from the ADC data to clean the signal from periodic contaminations (Fig. 2).

Comparison of graphs in [16] indicates that the LP approach can eliminate RFI narrow frequency contaminations as efficient as notch and FFT filters.

\section{ANALYSIS OF AERA EVENTS}

Figures 4 - 5 show spectra of original ADC traces and cleaned by the LP FIR filter with various values for parameter D of 128, 32 and 1, respectively (Fig. 3). For the mono-carrier contamination (Fig. 4) the RFI suppression is very good for any $\mathrm{D}$ parameter. For more structured spectral contaminations the suppression depends on the D parameter, nevertheless, we observe a reduction of periodic noise.

The delay-line $D$ implies that there is a gap between the samples that are used for the prediction and the sample that is to be predicted (Fig. 3). This delay-line is necessary to allow transient signals to pass through the filter unaltered. For $D=1$ the efficiency of the RFI suppression is maximal, however, the signal is significantly affected and a distortion factor (DF) (Fig. 6),

$$
D F=\sum_{k=-16}^{16}\left(1-\frac{\left(x_{F I R}\right)_{k}}{\left(x_{A D C}\right)_{k}}\right)^{2}
$$

introduced to estimate a quality of filtering, reaches a large, unacceptable value. For laboratory tests we selected $D=128$, to keep a reasonable safety margin in measurements (Fig. 7).

\section{LABORATORY TESTS}

The LP FIR filter has been tested with the Altera ${ }^{\circledR}$ DKDEV-5CEA7N Development Kit with a Cyclone ${ }^{\circledR}$ V FPGA and Texas Instr. ADS4249EVM Evaluation Module with 2channel 14-bits 250MSps ADC (ADS4249). Both modules were connected through the Altera ${ }^{\circledR}$ HSMC-ADC-BRIDGE providing the LVDS data transmission (Fig. 17).

At first the filter was tested by a mono-carrier drifting the frequency from $50.0 \mathrm{MHz}$ to $50.2 \mathrm{MHz}$ in $120 \mathrm{~s}$. The LP 


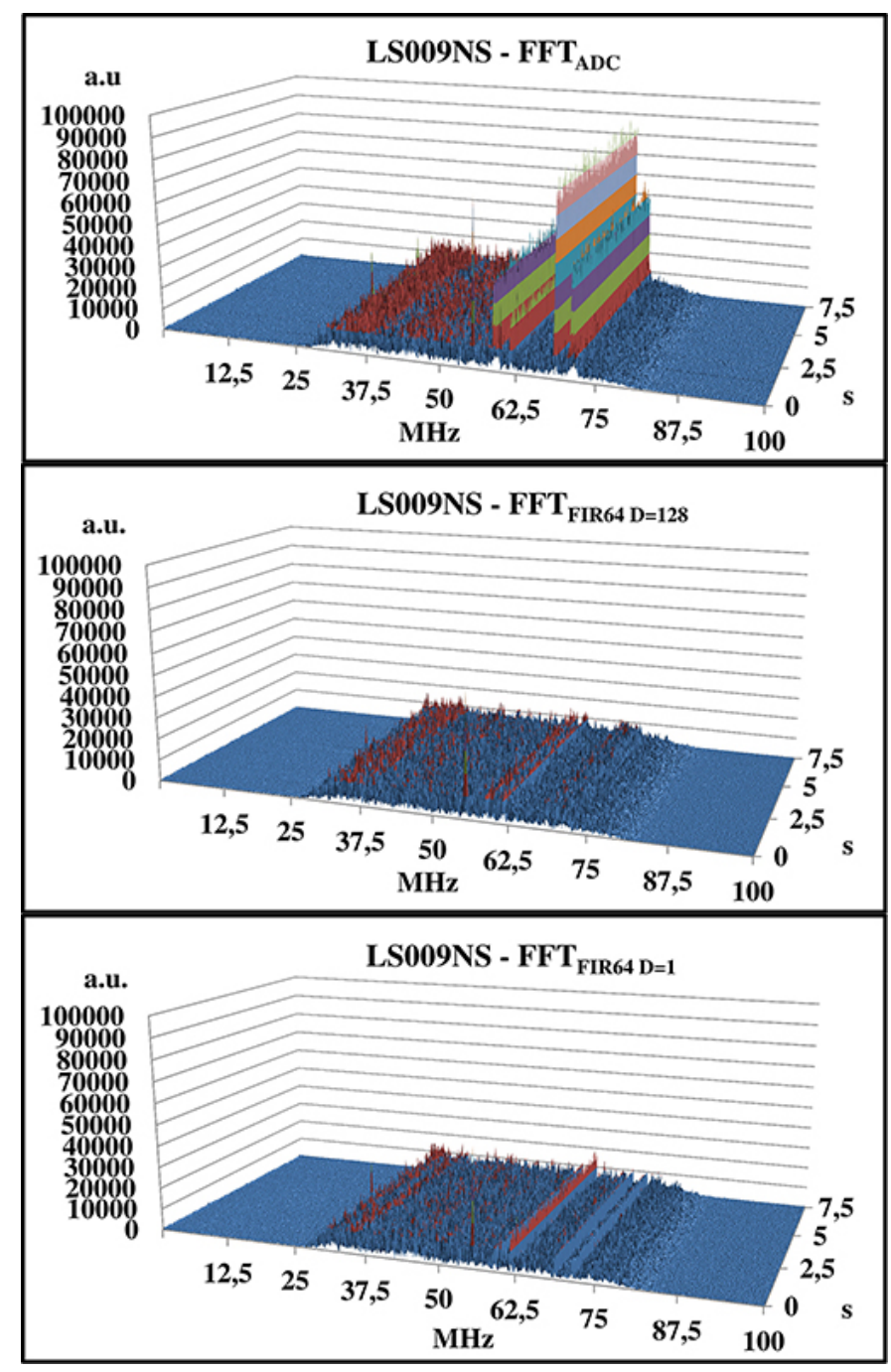

Fig. 4. FFT for ADC and FIR filter with various $\mathrm{D}=128$ and 1 , respectively, for long-term (7.5 s) data. Time $7.5 \mathrm{~s}$ corresponds to $1.5 \cdot 10^{9}$ cycles (sampling $=200 \mathrm{MSps}$ ). Registered events contain 1024 samples. The radio station LS009NS has been selected for a strong (100000 a.u. - arbitrary units) contamination by 4 carriers. The FIR64 filter shows a very good efficiency of the RFI suppression for all D factors (128 and 1).

coefficients were not calculated by $\mathrm{NIOS}^{\circledR}$ in a correlation with a generator running. Thus, these LP coefficients were used for the data cleaning in several tens of minutes (which corresponds to more than $10^{12}$ clock cycles). Fig. 8 shows a perfect long-term stability of the filter. If the generator driving the filter uses the frequency for which the LP coefficients were originally calculated, the suppression is almost total. Therefore, LP coefficients do not need to be refreshed very frequently.

Secondly, we checked the Hi-Fi FM configuration $(75 \mathrm{kHz}$ deviation of the $50 \mathrm{MHz}$ carrier with the maximal acoustic $15 \mathrm{kHz}$ modulation). This is very restrictive condition, which actually should not appear in real conditions in Argentina. The band $30-80 \mathrm{MHz}$ is used rather by narrow-band transmitter, while the FM Hi-Fi transmission is selected for the band of $88-108 \mathrm{MHz}$, cut-off by the band-pass analog filter. Nevertheless, even these critical contaminations were successfully suppressed (Fig. 9].
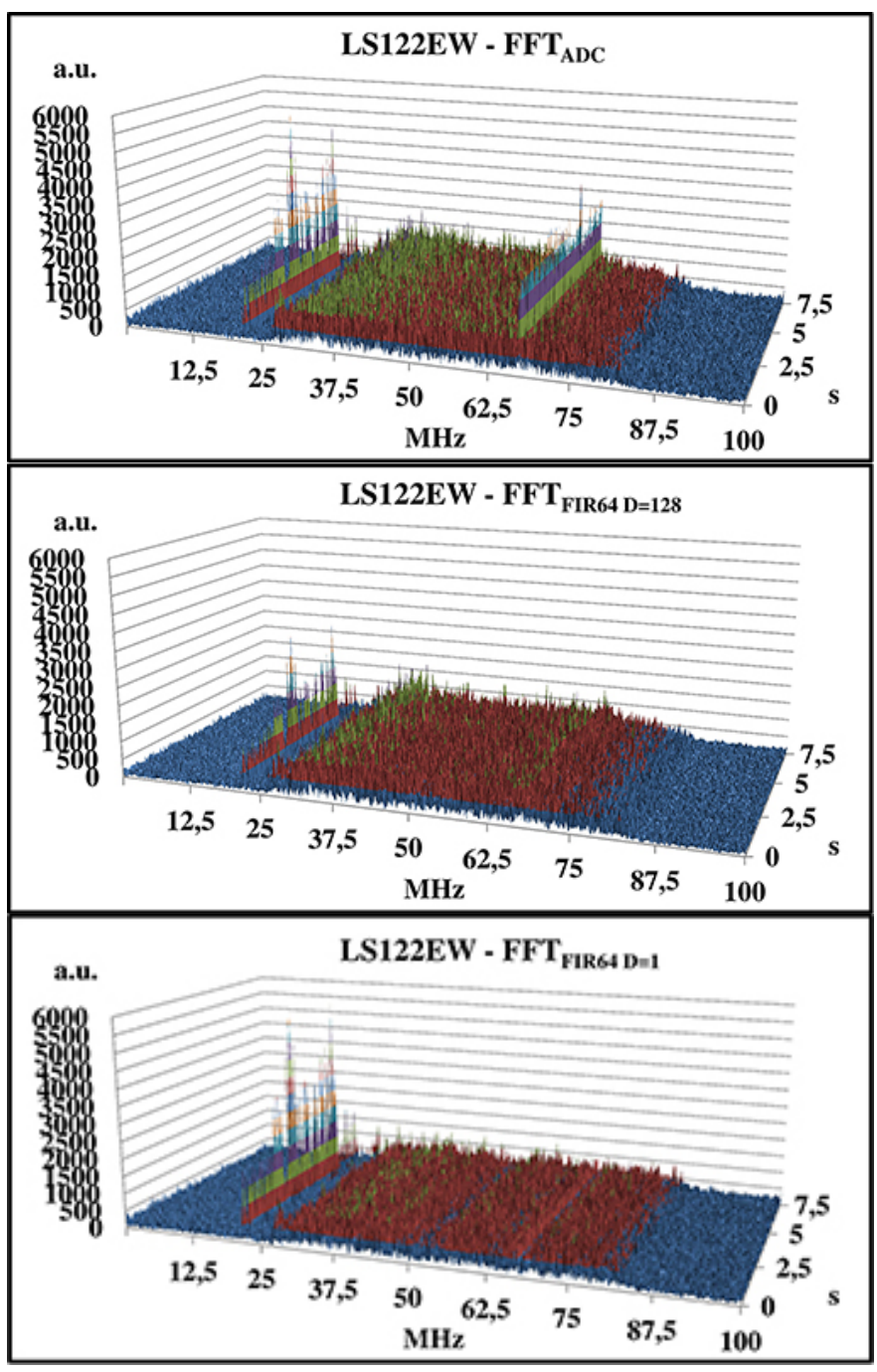

Fig. 5. Here, the radio station LS122EW has been selected for a weak (6000 a.u. - arbitrary units) contamination, focused on two narrow bands around 12 and $62 \mathrm{MHz}$. The suppression for $62 \mathrm{MHz}$ band is pretty good for all $\mathrm{D}$ factors, however for the $12 \mathrm{MHz}$ band, the RFI suppression is most efficient for $\mathrm{D}=128$.

We also tested a suppression efficiency (in the Fourier space) in the presence of relatively strong white noise (Fig. 11). Two pure carriers with 27.12 and $57.9 \mathrm{MHz}$ were wired mixed with noise. Fig. 10 shows that even when the noise level reaches the signal level the suppression factor remains on a level of 5-10 (for signals $50 \mathrm{mV}$ and $200 \mathrm{mV}$ contaminated by $50 \mathrm{mV}$ and $200 \mathrm{mV}$ of a noise). When the noise is small, the suppression factor reaches values up to 35 . However, a strong asymmetry is observed. The strong signal (i.e. $200 \mathrm{mV}$ of $27.12 \mathrm{MHz}$ ) is suppressed with a very high factor (35) while a 4 times smaller signal $(57.9 \mathrm{MHz})$ is almost not suppressed at all. For vice versa configuration a suppression structure remain the same.

\section{COMPARISON TO CURRENTLY USED IIR-NOTCH FILTER}

We compared suppression characteristics of the FIR filter based on the linear prediction with the currently used IIRnotch filter with 4 band-reject bands. Fig. 12 shows amplitudes of the $138^{\text {th }}$ frequency bin in the Fourier space corresponding 


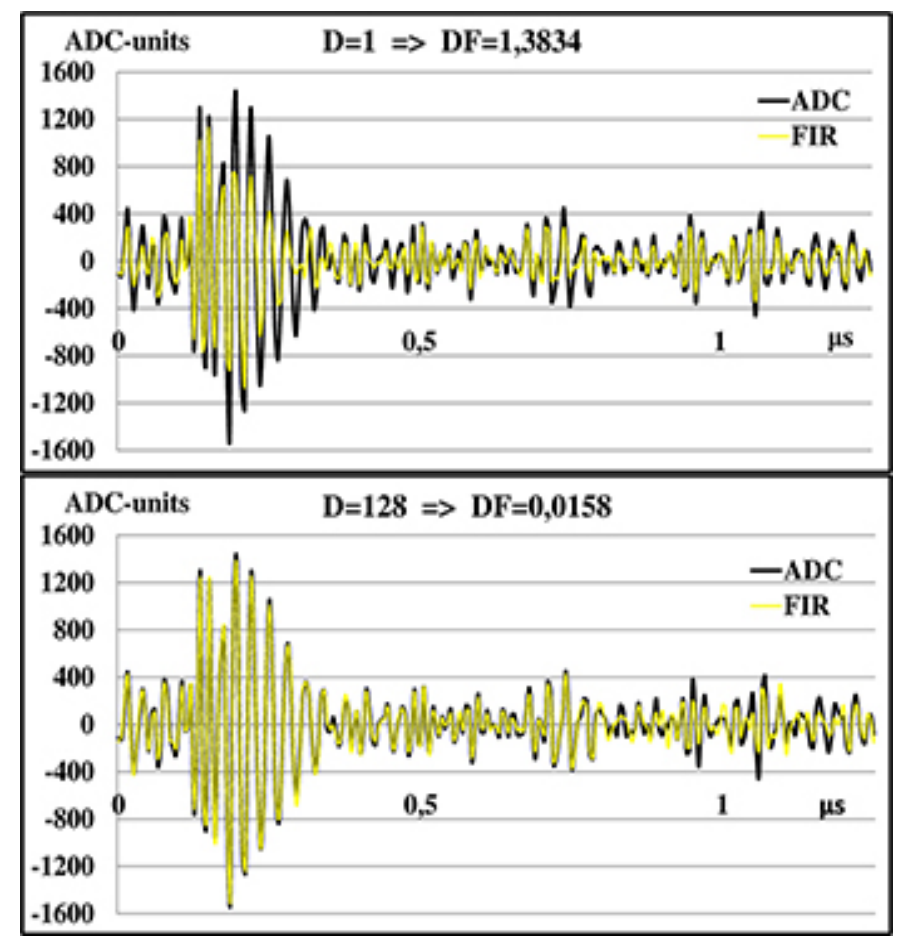

Fig. 6. Example of a potential signal distortion for $D=1$. It is recommended to introduce a delay for a signal modification. For $\mathrm{D}=128$ signals are almost unaffected.

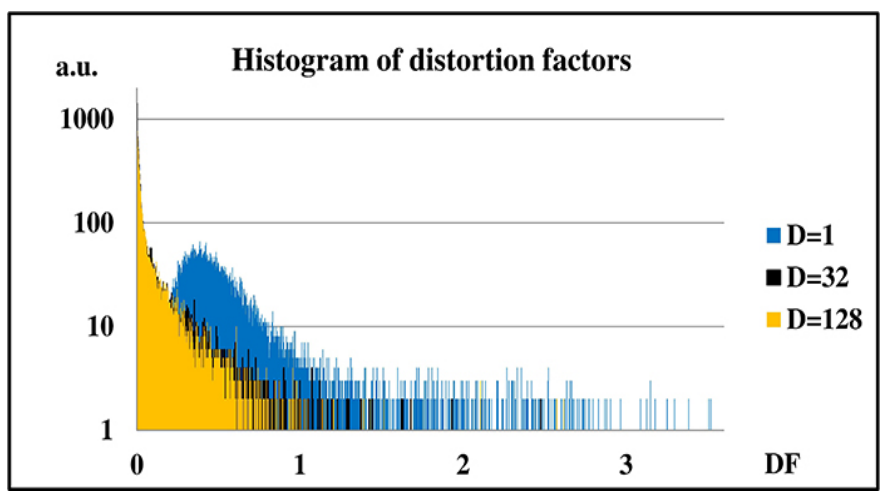

Fig. 7. Histogram of distortion factors (DF) for several hundreds AERA event filtered by the FIR filter with $\mathrm{D}=1,32$ and 128 , respectively. It is visible that a configuration with $\mathrm{D}=1$ distorts the signal too strong and cannot be used in a final design.

to a contribution of $1^{\text {st }}$ carrier contamination with a various frequencies in a range of $27.00-27.24 \mathrm{MHz}$ for a small (10 $\mathrm{mV}$ - upper graph) and significant $(100 \mathrm{mV}$ - lower graph) noise. It is well visible that the efficiency of the IIR filter is very high and, as expected, only around the reject frequency $(27.12 \mathrm{MHz}$ ) of the filter. Higher noise (comparison 100 $\mathrm{mV}$ vs. $10 \mathrm{mV}$ ) reduces the efficiency only slightly, thereby extending the width of the rejection band (from $\sim \pm 20 \mathrm{kHz}$ to $\sim \pm 30 \mathrm{kHz}$ ).

Fig. 13 shows the $283^{\text {th }}(55.2 \mathrm{MHz})$ and $296^{\text {th }}(57.9$ $\mathrm{MHz}$ ) Fourier modules vs. various frequencies in the $1^{\text {st }}$ channel contamination $(27.00-27.24 \mathrm{MHz})$. As expected, the suppression of the IIR filter outside the reject band is negligible. Note that for structured contaminations (two mono-

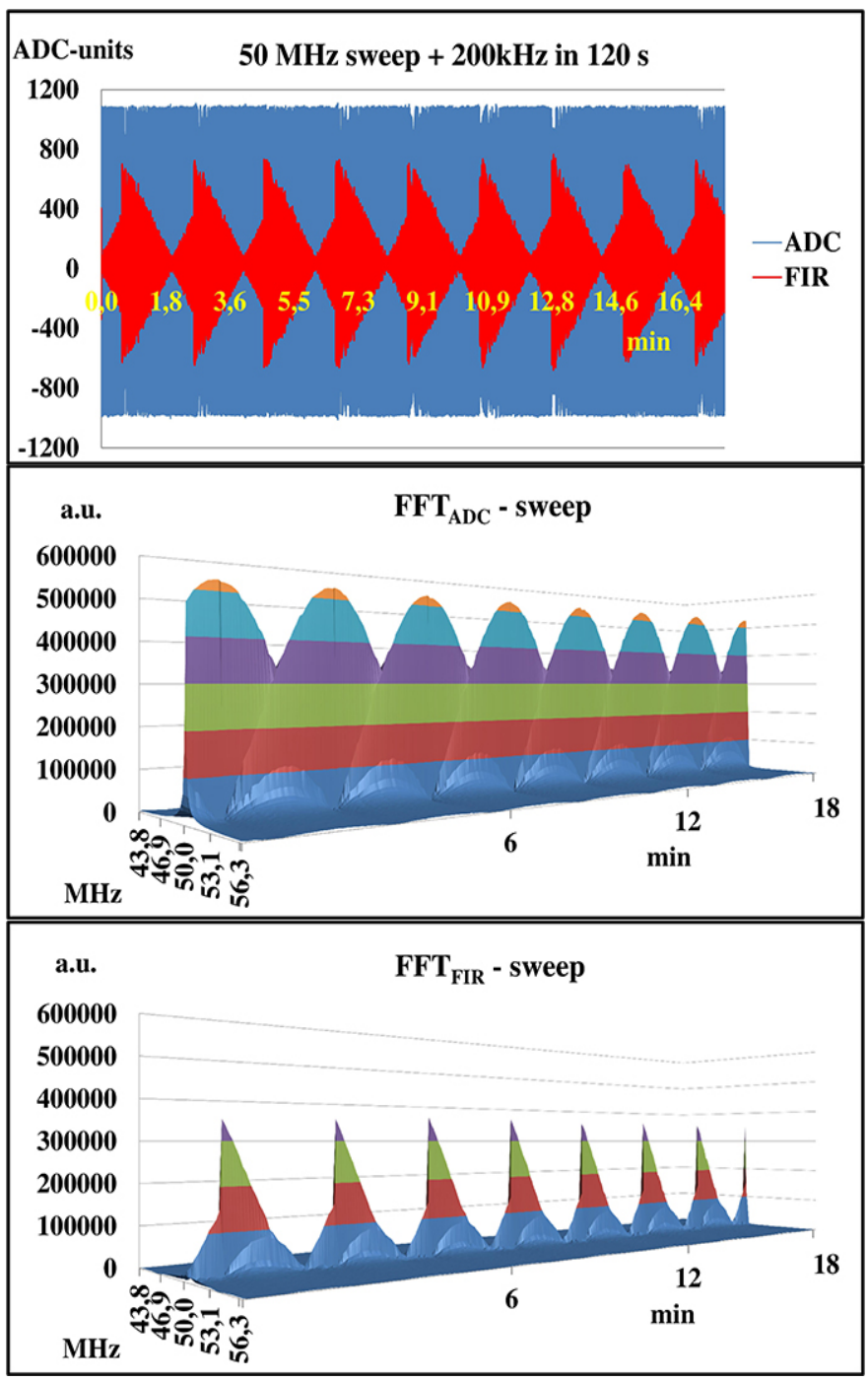

Fig. 8. Plots showing a suppression of the sine signal with a sweep from 50.0 $\mathrm{MHz}$ to $50.2 \mathrm{MHz}$ in 2 minutes. For 8 such a cycles we observe a perfect repetition. Plots obtained in real measurements on the laboratory setup for FIR64, $\mathrm{D}=128$ and 14-bit LP coefficients.

carriers with frequencies equal exactly the reject frequencies of the IIR-filter) the efficiency of the FIR filter is comparable with the efficiency of the IIR one, provided the background noise level is low in comparison to the contamination.

Figures 12 and 13 justify testing the FIR filter based on the linear predictor as an adaptive filter, adjusting the suppression characteristics to changing RFI conditions in the field.

\section{POWER CONSUMPTION}

A power consumption is the factor for systems supplied from solar panels. More sophisticated filter provides a better accuracy of data processing. However, the power efficiency may significantly decreased. We measured a power consumption for all developed FIR variants and compared with the currently used IIR filter. Fig 14 shows results for the current consumption with the NIOS processor (left panel) and with temporary blocked NIOS (right panel). The NIOS processor was used for the calculation of LP coefficients for the FIR 


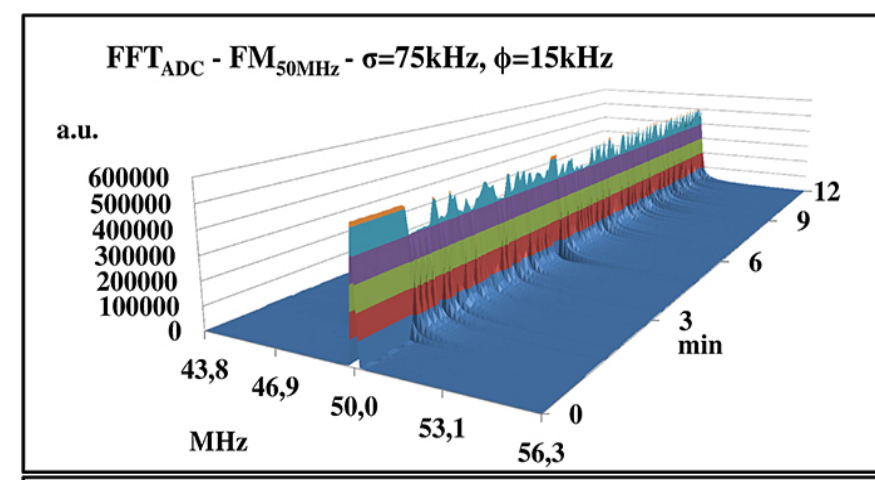

FFT $_{\mathrm{FIR}}-\mathrm{FM}_{50 \mathrm{MHz}}-\sigma=75 \mathrm{kHz}, \phi=15 \mathrm{kHz}$

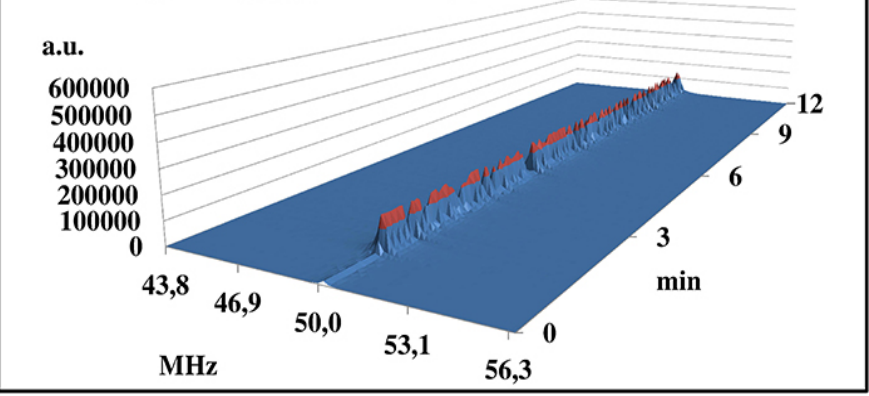

Fig. 9. Plots showing a suppression of the $50 \mathrm{MHz}$ signal frequency modulated (FM) with a deviation of $75 \mathrm{kHz}$ and a modulation of $15 \mathrm{kHz}$. The signal corresponds to Hi-Fi radio transmission. Even for wide-band FM the RFI suppression is relevant. Plots obtained in real measurements on the laboratory setup for FIR64, D=128 and 14-bit LP coefficients.

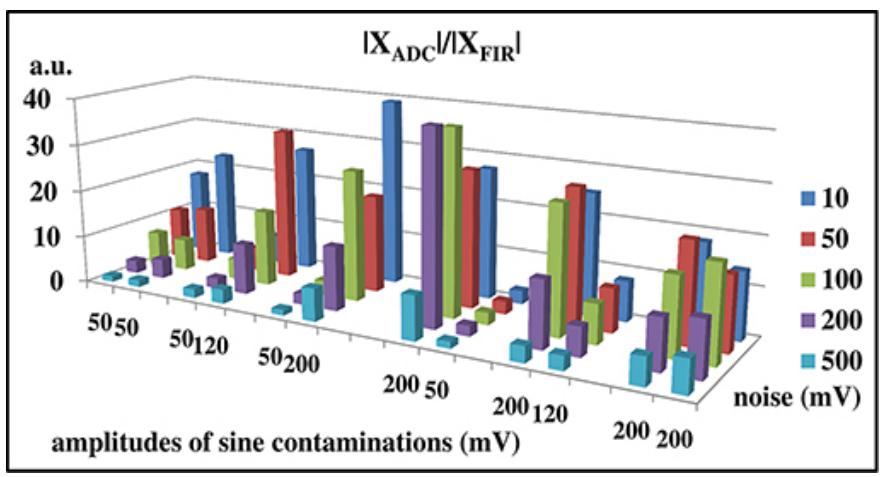

Fig. 10. Suppression factors for two sine pure carriers with frequencies 27.12 and $57.9 \mathrm{MHz}$ and $V_{p-p} 50$ and $200 \mathrm{mV}$, respectively, mixed with 10,50 , 100, 200 and $500 \mathrm{mV}$ noise.

filter and for data transmission via UART to the PC for both filters Fig 15 .

From Fig 14 it is visible that the FIR filter wit 32 stages and only 14-bit coefficients is at least power efficient in comparable to the IIR one. Longer FIR filters consume much more power and could be used exceptionally in extremely contaminated environment, where the suppression efficiency becomes the more important factor than the power efficiency.

\section{DATA FROM PAMPAS}

We have implemented the 32-stage LP filter (with $\mathrm{D}=128$ ) in the AERA radio station LS009. Fig. 16 show a suppression of the RFI in the higher frequency range. In comparison to Fig. 4 4 the suppression efficiency is lower, however, in current
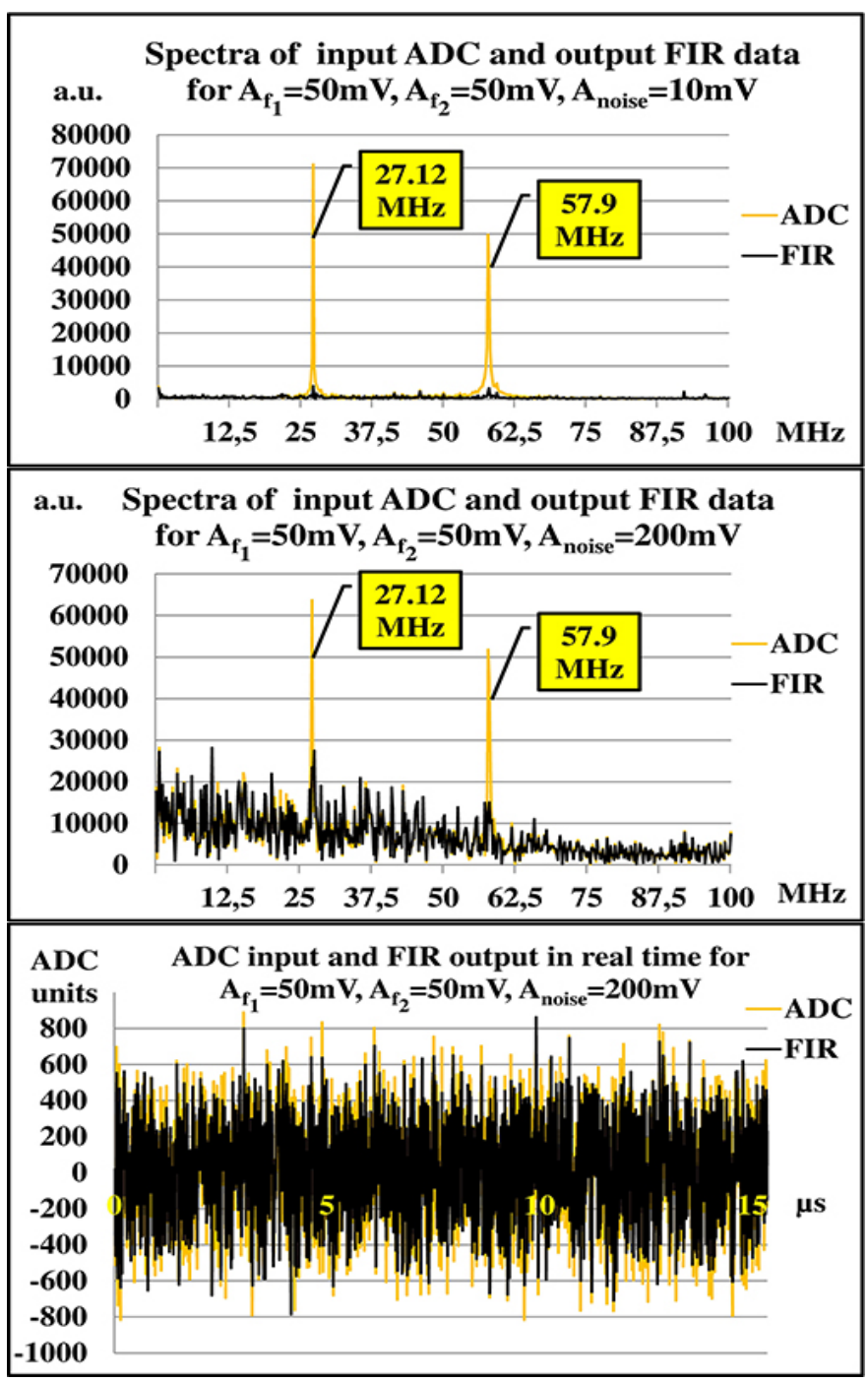

Fig. 11. Suppression factors for two sine pure carriers with frequencies 27.12 and $57.9 \mathrm{MHz}$ and $V_{p-p} 50$ and $200 \mathrm{mV}$, respectively, mixed with 10,50 , 100,200 and $500 \mathrm{mV}$ noise. Bottom graph shows real time traces for 200 $\mathrm{mV}$ noise. A suppression in the time domain is almost invisible

environment the huge contribution of non-stationary RFI was observed. In a presence of variable RFI the efficiency of the LP filter is not so high as for mono-carriers contamination. Fig. 16 shows that the noise suppression for the EW polarization is almost negligible.

\section{CONCLUSIONS}

Analytical calculations (Fig. 445) show that a very high efficiency of the LP filter can be obtained. The laboratory measurements confirmed that when the data is contaminated by mono-carriers, the suppression factor is very high. However, the environmental RFI as encountered in the Pierre Auger Observatory has a much more sophisticated structure. Therefore, the efficiency of the LP-filter significantly decreases.

Nevertheless, in each radio station the filter setup can be calculated, thereby optimizing the suppression factor, depending on the location of each station and the type of RFI contamination. 


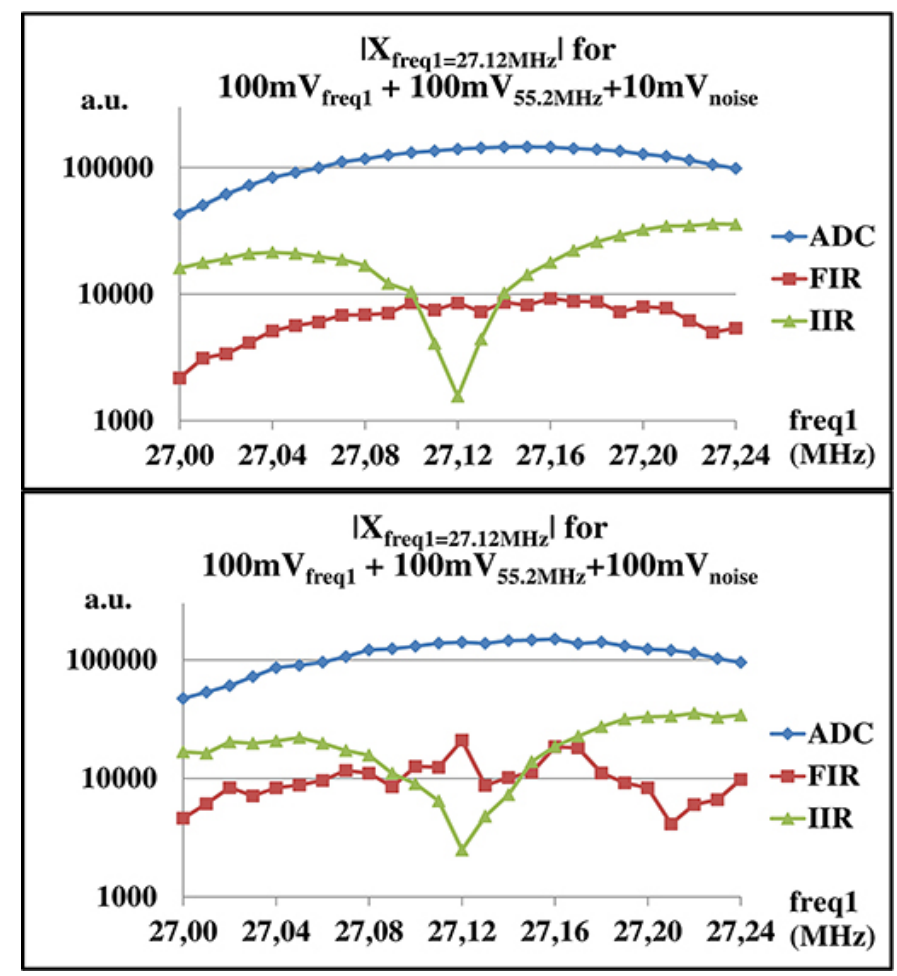

Fig. 12. Amplitudes of Fourier modules $\left(\bar{X}_{f r e q_{1}}=27.12 \mathrm{MHz}\right)$ for FIR filter based on the linear predictor and IIR-notch filter. The width of frequency bin is $\sim 0.1 \mathrm{MHz}$ (1024-point FFT), all $\bar{X}_{\text {freq }}$ contributions fall into $138^{t h}$ frequency bin. The signal with various frequencies freq $_{1}=27.00-27.24$ with a grid of $10 \mathrm{kHz}$ is provided by the $1^{\text {st }}$ channel of Tektronic AFG3252C, the $2^{\text {nd }}$ signal from the $2^{\text {nd }}$ channel of AFG3252C has a fixed frequency $=55.2$ $\mathrm{MHz}$, exactly equals the reject frequency of he $3^{\text {rd }}$ stage of the IIR-notch filter. The reject frequency of the $1^{\text {st }}$ IIR-not filter is $27.12 \mathrm{MHz}$. Additional $10 \mathrm{mV}$ or $100 \mathrm{mV}$ noise is provided by Agilent 33250A generator.

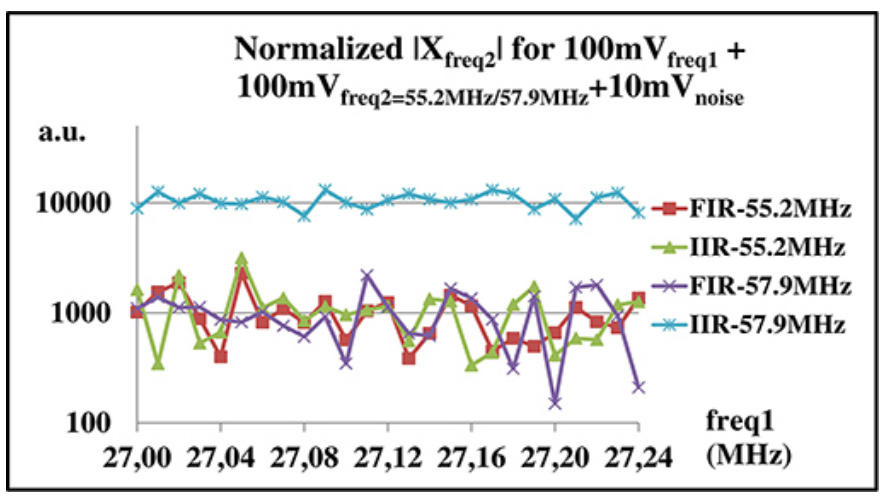

Fig. 13. Amplitudes of Fourier modules $\left(\bar{X}_{\text {freq }}=55.2 / 57.9 \mathrm{MHz}\right)$ for the $2^{\text {nd }}$ mono-carrier contamination with 55.2 or $55.9 \mathrm{MHz}$, respectively.

In AERA the Cyclone ${ }^{\circledR}$ IV FPGA EP4CE75F29I7 is currently in use. The laboratory tests provide input for an optimization of the RFI cleaning for the next generation of the AERA Front-End based on Cyclone ${ }^{\circledR} \mathrm{V}$ with Hardcore Processor System (HPS) and System on Chip (SoC).

\section{ACKNOWLEDGMENT}

This work was supported by the Polish National Center for Research and Development under NCBiR Grant No. ERA/NET/ASPERA/02/11, by the National Science Centre

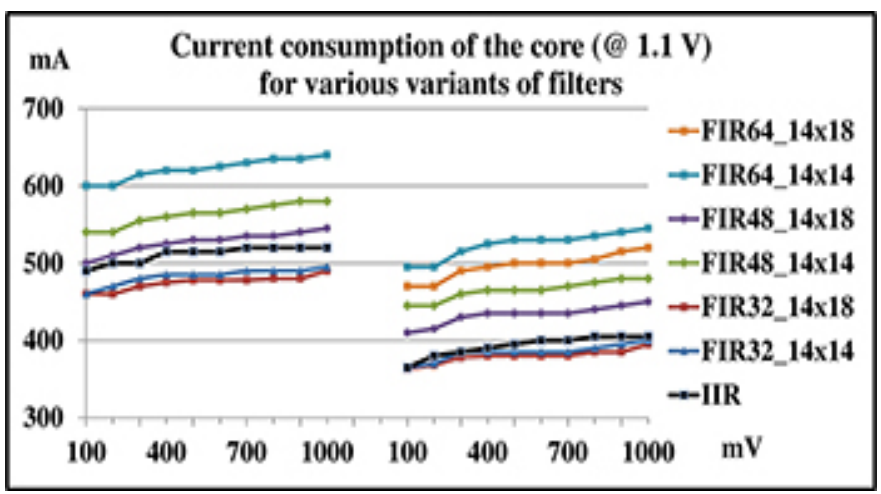

Fig. 14. The current consumption for several tested variants of the FIR filter with a comparison to the currently used IIR one.

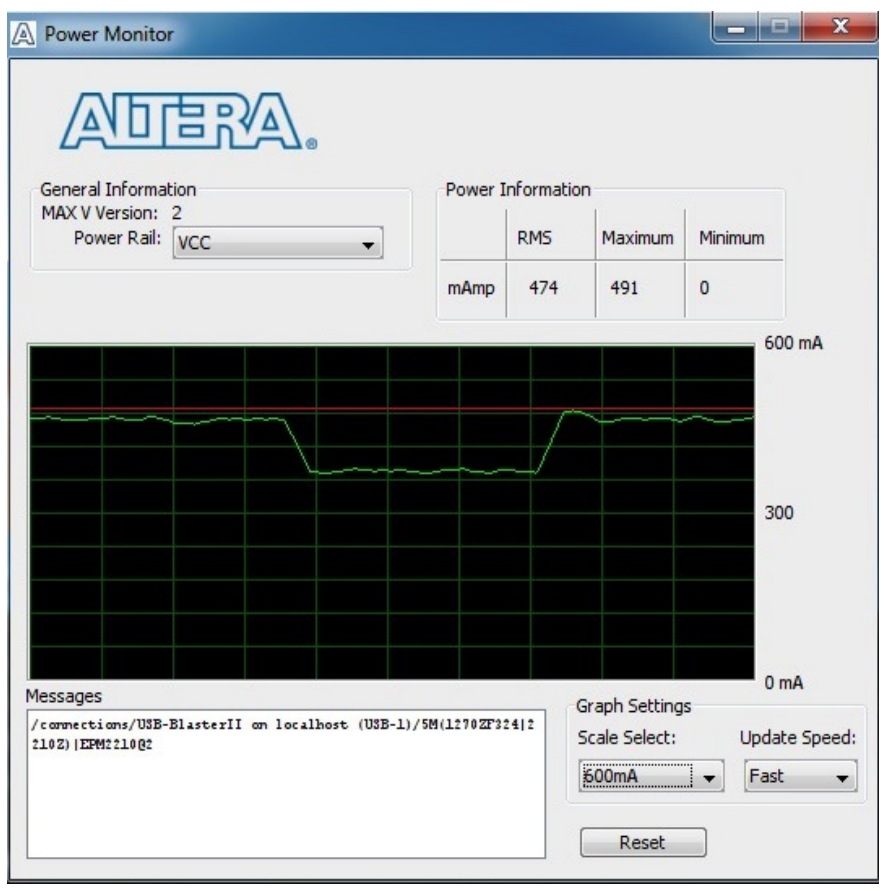

Fig. 15. A graph showing the current consumption of the core (@1.1 V). A reduction of the current consumption corresponds to temporary disabling of the NIOS processor

(Poland) under NCN Grant No. 2013/08/M/ST9/00322, , and by the Ministerie van Onderwijs, Cultuur en Wetenschap, Nederlandse Organisatie voor Wetenschappelijk Onderzoek (NWO), Stichting voor Fundamenteel Onderzoek der Materie (FOM), the Netherlands.

\section{REFERENCES}

[1] H. Falcke,W. D. Apel, A. F. Badea, et al., "Detection and imaging of atmospheric radio flashes from cosmic ray air showers", Nature, vol. 435, pp. 313-316, May 2005.

[2] D. Ardouin, A. Belletoile, D. Charrier, et al., "Radioelectric field features of extensive air showers observed with CODALEMA" Astropart. Phys., vol. 26, pp. 341-350, Dec. 2006

[3] S. Fliescher for the Pierre Auger Collaboration, "Radio detection of cosmic ray induced air showers at the Pierre Auger Observatory", Nucl. Instr. Meth., ser. A, vol. 662, pp. 124-129, Jan. 2012.

[4] J. Abraham et al., [Pierre Auger Collaboration], "Properties and Performance of the Prototype Instrument for the Pierre Auger Observatory", Nucl. Instr. Meth., ser. A, vol. 523, pp. 50-95, May 2004. 


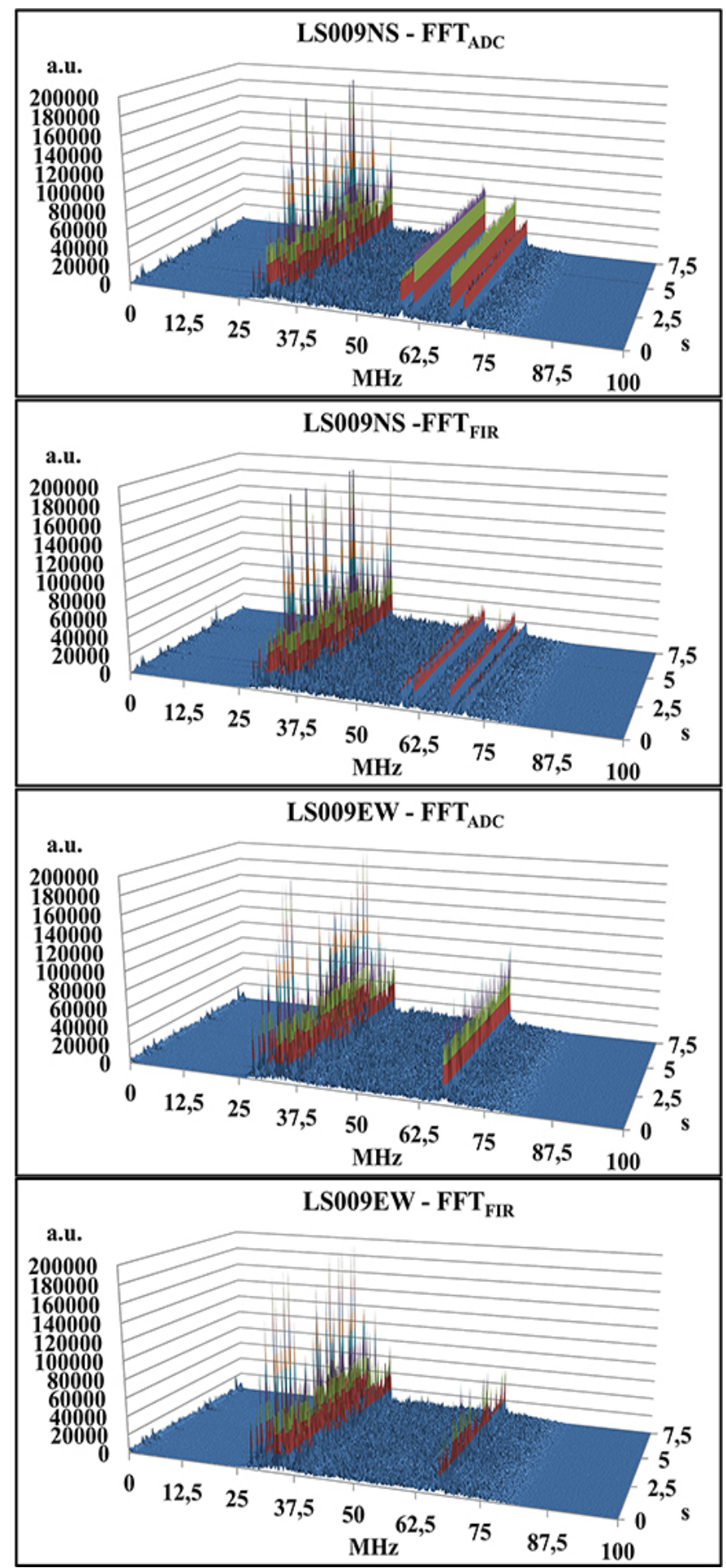

Fig. 16. FFT for ADC and FIR filter with $\mathrm{D}=128$, for long-term $(7.5 \mathrm{~s})$ data for the radio station LS009 and NS and EW polarizations. As for Fig. 4 we see 4 mono-carriers, however with additional low frequency non-stationary RFI. The presence of the variable in time RFI reduces an efficiency of the LP filter.

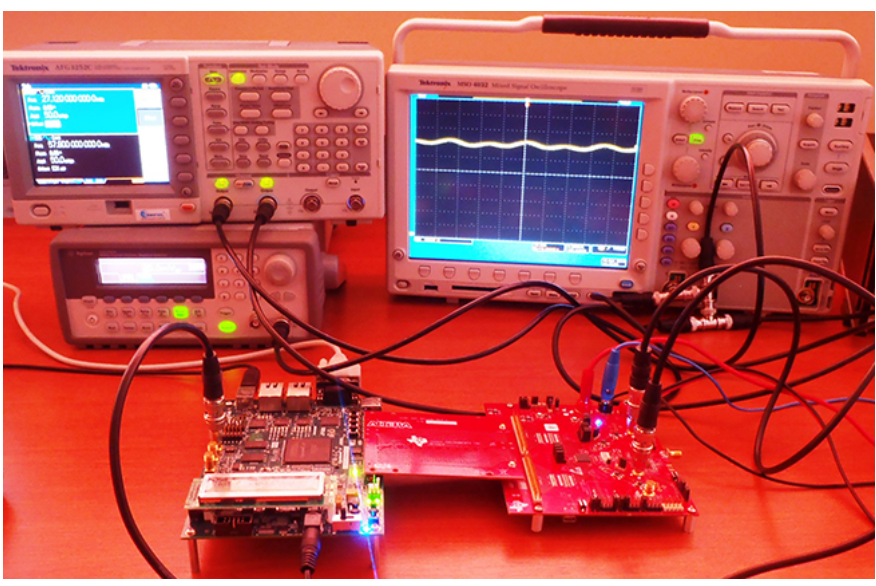

Fig. 17. The laboratory measurement setup with the Altera ${ }^{\circledR}$ DKDEV-5CEA7N Development Kit with Cyclone ${ }^{\circledR}$ V FPGA and Texas Instr. ADS4249EVM Evaluation Module with 2-channel 14-bits 250MSps ADC (ADS4249). Both modules were connected by the Altera ${ }^{\circledR}$ HSMC-ADCBRIDGE providing the LVDS data transmission. Tektronix AFG3252C 2channel generator provides 2 sine waveforms, Agilent 33250A provides noise signal.

[5] D. J. Fegan, "Detection of elusive radio and optical emission from cosmicray showers in the 1960s", Nucl. Instr. Meth., ser. A, vol. 662, pp. 2-11, Jan. 2012.

[6] F. D. Kahn and I. Lerche, "Radiation from Cosmic Ray Air Showers", Proc. Roy. Soc. A, vol. 289, pp. 206-213, Jan. 1966.

[7] G. A. Askaryan, Journal of Exp. and Theoretical Phys., vol. 14, pp. 441, 1962.

[8] G. A. Askaryan, "Coherent Radio Emission from Cosmic Showers in Air and in Dense Media", Journal of Exp. and Theoretical Phys., vol. 21, pp. 658-659, Jan. 1965.

[9] A. Aab et al., "Probing the radio emission from air showers with polarization measurements", Phys. Rev. D89, 052002, Mar. 2014.

[10] G. A. Dulk, W. C. Erickson, R. Manning, and J.-L. Bougeret, "Calibration of low-frequency radio telescopes using the galactic background radiation", $A \& A$, vol. 365, pp. 294-300, Jan. 2001

[11] K. K. Parhi and D. G. Messerschmitt, IEEE Trans. on Acoustics, Speech, and Signal Processing, vol. 37, pp. 1099-1117, 1989.

[12] J. Makhoul, "Linear prediction: A tutorial review" Proc. of the IEEE, vol. 63, no. 4, pp. 561-580, Apr. 1975.

[13] D. Li, D. O'Shaughnessy, "Speech processing: a dynamic and optimization-oriented approach", pp. 4148. ISBN 0-8247-4040-8 (2003)

[14] Z. Szadkowski, E.D. Fraenkel, A. M. van den Berg, "FPGA/NIOS Implementation of an Adaptive FIR Filter Using Linear Prediction to Reduce Narrow-Band RFI for Radio Detection of Cosmic Rays", IEEE Trans. on Nucl. Science, vol. 60, pp. 3483-3490, Oct. 2013.

[15] Z. Szadkowski, E.D. Fraenkel, D. Głas, R. Legumina, "An optimization of the FPGA/NIOS adaptive FIR filter using linear prediction to reduce narrow band RFI for the next generation ground-based ultra-high energy cosmic-ray experiment", Nucl. Instr. Meth., ser. A, vol. 732, pp. 535-539, June 2013.

[16] Z. Szadkowski, Ad M. van den Berg, E.D. Fraenkel, D. Głas, J. Kelley, C. Timmermans, T. Wijnen, "Analysis of the efficiency of the filters suppressing the RFI being developed for the extension of AERA", 33nd International Cosmic Ray Conference - July 2013 - Rio de Janeiro, Brazil. 